\begin{tabular}{|c|c|}
\hline Title & Simple matrix representations of the orthogonal polynomials for a rational spectral density on the unit circle \\
\hline Author(s) & Inoue, A kihiko; Kasahara, Y ukio \\
\hline Citation & $\begin{array}{l}\text { Journal of mathematical analysis and applications, 464(2), 1366-1374 } \\
\text { https://doi.org/10.1016/.jmaa.2018.04.062 }\end{array}$ \\
\hline Issue Date & $2018-08-15$ \\
\hline Doc URL & http:/hdl .handle.net/2115/79065 \\
\hline Rights & $\begin{array}{l}\text { (O2018. Ins manuscript versson is made avallable under the CC-BY-IVC-IVD } 4.0 \text { IIcense } \\
\text { http://treativecommons.org/icenses/py-nc-nd/4.0/ }\end{array}$ \\
\hline Rights(URL) & http://creativecommons.org/icenses/by-nc-nd/4.0/ \\
\hline Type & article (author version) \\
\hline File Information & IK-JMAA-rev.pdf \\
\hline
\end{tabular}

Instructions for use 


\title{
Simple matrix representations of the orthogonal polynomials for a rational spectral density on the unit circle
}

\author{
Akihiko Inoue $^{\mathrm{a}}$, Yukio Kasahara ${ }^{\mathrm{b}, *}$ \\ ${ }^{a}$ Department of Mathematics, Hiroshima University, Higashi-Hiroshima, 739-8526, Japan \\ ${ }^{b}$ Department of Mathematics, Hokkaido University, Sapporo, 060-0810, Japan
}

\begin{abstract}
In this note, by using a discrete analog of a projection formula introduced by A. Seghier in 1978, we calculate the orthogonal polynomials on the unit circle for a rational spectral density having no zeros there, and derive simple matrix representations of themselves, their squared norms, and the Verblunsky coefficients.
\end{abstract}

Keywords: Orthogonal polynomials on the unit circle, Verblunsky coefficients, rational spectral density, Seghier's formula

MSC: 42C05, 60G10, 60G25.

\section{Introduction}

Let $\mu$ be a probability measure on the unit circle $\mathbb{T}$ that is supported on an infinite set. By applying the Gram-Schmidt method to the sequence of monomials $1, z, z^{2}, \ldots$ in the Hilbert space $L^{2}(\mu)$ with

$$
(\varphi, \psi)_{\mu}=\int_{0}^{2 \pi} \varphi\left(e^{i \theta}\right) \overline{\psi\left(e^{i \theta}\right)} d \mu(\theta), \quad\|\varphi\|_{\mu}=\left(\int_{0}^{2 \pi}\left|\varphi\left(e^{i \theta}\right)\right|^{2} d \mu(\theta)\right)^{1 / 2}
$$

one obtains a sequence of the monic orthogonal polynomials $\Phi_{0}, \Phi_{1}, \Phi_{2}, \ldots$ with respect to $\mu$, i.e.,

$$
\Phi_{n}(z)=z^{n}+\text { lower order, } \quad\left(\Phi_{n}, \Phi_{n^{\prime}}\right)_{\mu}=0 \quad\left(n \neq n^{\prime}\right) .
$$

Their constant terms $\alpha_{n}=\Phi_{n}(0)$ lie in the open unit disc $\mathbb{D}$ for all $n=1,2, \ldots$ Therefore, $\mu$ yields a sequence $\left\{\alpha_{1}, \alpha_{2}, \ldots\right\}$ in $\mathbb{D}$. Verblunsky's theorem states that every sequence in $\mathbb{D}$ arises in this way from a unique probability measure on $\mathbb{T}$ that is supported on an infinite set, whence $\alpha_{n}$ are called the Verblunsky coefficients. (There are several other names such as the Schur parameters.) As an application, the monic orthogonal polynomials are used for studying the prediction problem for a centered, complexvalued stationary time series $\left\{X_{k}\right\}$ with spectral measure $\mu$, i.e.,

$$
\operatorname{Cov}\left(X_{j}, X_{k}\right)=\int_{0}^{2 \pi} e^{i(j-k) \theta} d \mu(\theta) \quad(j, k=0, \pm 1, \pm 2, \ldots) .
$$

More precisely, $\Phi_{n}$ is a spectral counterpart of the one-step prediction error in the sense that

$$
X_{n}-\hat{X}_{n}=X_{n}-\sum_{k=1}^{n} \phi_{n, k} X_{n-k} \quad \leftrightarrow \quad \Phi_{n}(z)=z^{n}-\sum_{k=1}^{n} \phi_{n, k} z^{n-k}, \quad \operatorname{Var}\left(X_{n}-\hat{X}_{n}\right)=\left\|\Phi_{n}\right\|_{\mu}^{2},
$$

\footnotetext{
* Corresponding author

Email addresses: inoue100@hiroshima-u.ac.jp (Akihiko Inoue), y-kasa@math.sci.hokudai.ac.jp (Yukio Kasahara)
} 
where $\hat{X}_{n}$ is the linear predictor of $X_{n}$ based on $\left\{X_{0}, X_{1}, \ldots, X_{n-1}\right\}$. Since $\phi_{n, n}=-\alpha_{n}$, the Verblunsky coefficients are essentially the same as the partial autocorrelation function $\left\{\phi_{n, n}\right\}$. See Simon [11] for the theory of orthogonal polynomials on the unit circle, and Brockwell-Davis [2] for the prediction problem of stationary time series.

Inoue $[3,4,5]$, Inoue-Kasahara [6, 7] and Bingham et al. [1] have developed explicit representations of the predictor coefficients and prediction error variances, and studied their applications. Taking a different approach, this note presents simple matrix representations of the monic orthogonal polynomials and related quantities for a probability measure of the form $d \mu(\theta)=w\left(e^{i \theta}\right) \frac{d \theta}{2 \pi}$, where $w$ is a rational function of $e^{i \theta}$ satisfying $w\left(e^{i \theta}\right) \neq 0$ on $\mathbb{T}$. As is well known, the latter can be expressed as

$$
w\left(e^{i \theta}\right)=\frac{\left|P\left(e^{i \theta}\right)\right|^{2}}{\left|Q\left(e^{i \theta}\right)\right|^{2}}
$$

with some polynomials $P$ and $Q$ which have no common zeros and satisfy

$$
P(0)>0, \quad Q(0)>0, \quad P(z) \neq 0, \quad Q(z) \neq 0 \quad(z \in \mathbb{D} \cup \mathbb{T}) .
$$

Such a rational function is important in time series analysis since it is the spectral density of a causal and invertible ARMA (autoregressive-moving average) process, characterized by the difference equation

$$
Q(B) X_{k}=P(B) Z_{k} \quad(k=0, \pm 1, \pm 2, \ldots),
$$

where $B$ is the backward shift operator $\left(B X_{k}=X_{k-1}\right)$ and $\left\{Z_{k}\right\}$ is a white noise $\left(\operatorname{Cov}\left(Z_{j}, Z_{k}\right)=\delta_{j k}\right)$. The matrix representations are derived by calculating $\Phi_{n}$ via an analog of a projection formula introduced by Seghier [10], which is explained in Section 2. The representations themselves, including those of the squared norms $\left\|\Phi_{n}\right\|_{\mu}^{2}$ and the Verblunsky coefficients $\alpha_{n}$, are given in Section 3. Among other results, the representation of $\alpha_{n}$ is particularly simple.

We dedicate this note to Yusuke Nakamura (1992-2016) who discovered a similar matrix representation of the finite predictor for some continuous-time process, in joint work with the first author. It was this discovery that made the latter realize, after his death, the existence of explicit formulas in a simple case of this paper, and thus led us to the present work.

\section{Seghier's formula}

In his work on the prediction problem for a continuous-time stationary process, Seghier [10] introduced a projection formula which is useful when the process has a rational spectral density. This section is devoted to a brief discussion on its discrete analog and related matters. For details, see Kasahara-Bingham [8].

Let $\mathbb{D}, \mathbb{T}$ and $\mathbb{E}$ denote the open unit disc, the unit circle, and the region outside the circle in the extended complex plane, respectively, so that $\mathbb{C} \cup\{\infty\}=\mathbb{D} \cup \mathbb{T} \cup \mathbb{E}$ (disjoint union). Recall the Hilbert space $L^{2}(\mu)$ from Section 1. Let $H^{2}(\mu)$ and $K^{2}(\mu)$ be the closed subspaces of $L^{2}(\mu)$ spanned by $\left\{1, e^{i \theta}, e^{2 i \theta}, \ldots\right\}$ and $\left\{e^{-i \theta}, e^{-2 i \theta}, \ldots\right\}$, respectively. Write $L^{2}, H^{2}$ and $K^{2}$ for these spaces with the normalized Lebesgue measure $d \mu(\theta)=\frac{d \theta}{2 \pi}$, which gives $L^{2}=H^{2} \oplus K^{2}$ (orthogonal decomposition). The functions in $H^{2}$ and $K^{2}$ have natural analytic extensions into $\mathbb{D}$ and $\mathbb{E}$, respectively. Denote by $\mathbb{C}\left[e^{i \theta}\right]_{<n}$ the space of polynomials of $e^{i \theta}$ with degree less than $n$. The monic orthogonal polynomial $\Phi_{n}$ is the projection of $e^{i n \theta}$ onto the orthogonal complement of $\mathbb{C}\left[e^{i \theta}\right]_{<n}$ in $L^{2}(\mu)$.

Let $d \mu(\theta)=w\left(e^{i \theta}\right) \frac{d \theta}{2 \pi}+d \mu_{s}(\theta)$, where $\mu_{s}$ is the singular part. If $\log w$ is integrable, there exists a unique outer function $h$, called the Szegö function, in $H^{2}$ such that $w=|h|^{2}$ and $h(0)>0$. By [8, Theorem 3.1], the equation

$$
H^{2}(\mu) \cap e^{i n \theta} K^{2}(\mu)=\mathbb{C}\left[e^{i \theta}\right]_{<n}
$$

holds for every $n=0,1,2, \ldots$ if and only if $\mu_{s}=0, \log w$ is integrable, and $h^{2}$ is rigid, which means that, except for its positive constant multiples, no functions in the classical Hardy space $H^{1}$ share with it the same argument. Set

$$
\phi^{\dagger}(z)=\overline{\phi(1 / \bar{z})}
$$


For $n=0,1,2, \ldots$, let $H_{n}$ be the Hankel operator with symbol $e^{i n \theta}\left(h / h^{\dagger}\right)$ and $H_{n}^{*}$ its adjoint operator, i.e.,

$$
H_{n}(\varphi)=\mathscr{P}_{K^{2}}\left\{e^{i n \theta}\left(h / h^{\dagger}\right) \varphi\right\} \quad\left(\varphi \in H^{2}\right), \quad H_{n}^{*}(\psi)=\mathscr{P}_{H^{2}}\left\{e^{-i n \theta}\left(h^{\dagger} / h\right) \psi\right\} \quad\left(\psi \in K^{2}\right),
$$

where $\mathscr{P}_{S}$ is the orthogonal projection operator of $L^{2}$ onto $S$. By [8, Remark 3.4 and Proposition 3.5], if (3) holds and also if $I-H_{n}^{*} H_{n}$ is invertible, the monic orthogonal polynomial $\Phi_{n}$ can be expressed as

$$
\Phi_{n}=h(0)\left\{e^{i n \theta}\left(h^{\dagger}\right)^{-1}\left(I-H_{n}^{*} H_{n}\right)^{-1} 1-h^{-1} H_{n}\left(I-H_{n}^{*} H_{n}\right)^{-1} 1\right\},
$$

which is a discrete analog of Seghier's projection formula in [10, Proposition 3].

\section{Results}

We calculate the monic orthogonal polynomials for a rational spectral density having no zeros on the unit circle, and derive matrix representations of themselves and the related quantities.

Throughout this section, assume that $\mu_{s}=0$ and $w$ is a rational function of $e^{i \theta}$ having no zeros on $\mathbb{T}$. In this case, $h^{2}$ is rigid since its reciprocal lies in $H^{1}$; see Yabuta [12]. Also, $H_{n}$ is strictly contractive for all $n=0,1,2, \ldots$ since the Helson-Szegö theorem implies $\left\|H_{0}\right\|<1$, and also $\left\|H_{n}\right\|$ is non-increasing in $n$; see Peller [9, Chapter 9]. (The value of $\left\|H_{n}\right\|$ is equal to the cosine of the angle between "past" $K^{2}(\nu)$ and "future" $e^{i n \theta} H^{2}(\nu)$, where $d \nu=w^{-1} \frac{d \theta}{2 \pi}$.) Consequently, (3) holds true, $I-H_{n}^{*} H_{n}$ is invertible, and thus, Seghier's formula (4) is available for all $n=0,1,2, \ldots$

Let $P$ and $Q$ be the polynomials satisfying (1) and (2). Without loss of generality, one may assume that $P(0)=1$, and also that $P$ is non-constant. (For, if $P(z)=1$, then $\Phi_{n}(z)=z^{n} Q^{\dagger}(z)$ for $n \geq \operatorname{deg} Q$, and $\alpha_{n}=0$ for $n>\operatorname{deg} Q$, hence there is nothing to do; see [2, Example 3.4.3] and [11, Corollary 1.7.6].) This assumption permits us to write

$$
P(z)=\prod_{i=1}^{d}\left(1-\bar{p}_{i} z\right)^{m_{i}}
$$

where $p_{1}, p_{2}, \ldots, p_{d}$ are distinct points in $\mathbb{D} \backslash\{0\}$ and $m_{1}, m_{2}, \ldots, m_{d}$ are positive integers. Define

$$
m=\operatorname{deg} P\left(=m_{1}+m_{2}+\cdots+m_{d}\right), \quad n_{0}=\max \{\operatorname{deg} Q-\operatorname{deg} P, 0\} .
$$

By the conditions (1) and (2), the Szegö function $h$ is given by

$$
h(z)=\frac{P(z)}{Q(z)}
$$

Since

$$
\frac{1}{h^{\dagger}(z)}=\frac{z^{m} Q^{\dagger}(z)}{\prod_{i=1}^{d}\left(z-p_{i}\right)^{m_{i}}}
$$

one may write

$$
\frac{h(z)}{h^{\dagger}(z)}=\sum_{i=1}^{d} \sum_{j=1}^{m_{i}} \frac{a_{i j}}{\left(z-p_{i}\right)^{j}}+R(z),
$$

where $a_{i j}$ is the $j$-th coefficient in the principal part of the Laurent series expansion at a pole $z=p_{i}$, i.e.,

$$
a_{i j}=\lim _{z \rightarrow p_{i}} \frac{1}{\left(m_{i}-j\right) !} \frac{d^{m_{i}-j}}{d z^{m_{i}-j}} \frac{\left(z-p_{i}\right)^{m_{i}} h(z)}{h^{\dagger}(z)}
$$

and $R$ is a rational function which is holomorphic in $\mathbb{D} \cup \mathbb{T}$ except for a pole at $z=0$ of multiplicity $n_{0}$ in the case $n_{0}>0$. Set

$$
\phi^{\sharp}(z)=(1 / z) \overline{\phi(1 / \bar{z})},
$$


which provides us with

$$
\left(\phi^{\sharp}\right)^{\sharp}=\phi, \quad H^{2} \ni \varphi \mapsto \varphi^{\sharp} \in K^{2}, \quad K^{2} \ni \psi \mapsto \psi^{\sharp} \in H^{2}, \quad H_{n}^{*} \psi=\left(H_{n} \psi^{\sharp}\right)^{\sharp} .
$$

To check the last equation, use $\left(\mathscr{P}_{H^{2}} \phi\right)^{\sharp}=\mathscr{P}_{K^{2}}\left(\phi^{\sharp}\right)$ and $(\chi \psi)^{\sharp}=\chi^{\dagger} \psi^{\sharp}$ in the following way:

$$
\left[\mathscr{P}_{H^{2}}\left\{e^{-i n \theta}\left(h^{\dagger} / h\right) \psi\right\}\right]^{\sharp}=\mathscr{P}_{K^{2}}\left[\left\{e^{-i n \theta}\left(h^{\dagger} / h\right) \psi\right\}^{\sharp}\right]=\mathscr{P}_{K^{2}}\left[\left\{e^{-i n \theta}\left(h^{\dagger} / h\right)\right\}^{\dagger} \psi^{\sharp}\right]=\mathscr{P}_{K^{2}}\left\{e^{i n \theta}\left(h / h^{\dagger}\right) \psi^{\sharp}\right\} .
$$

As will be seen presently, the Hankel operators $H_{n}$ are closely related with the rational functions

$$
f_{i j}(z)=\frac{z^{j-1}}{\left(1-\bar{p}_{i} z\right)^{j}}, \quad f_{i j}^{\sharp}(z)=\frac{1}{\left(z-p_{i}\right)^{j}} .
$$

Define a vector-valued function $\boldsymbol{f}$ by the $\left(m_{1}, m_{2}, \ldots, m_{d}\right)$-block representation

$$
\boldsymbol{f}(z)={ }^{T}\left(f_{11}(z), \ldots, f_{1 m_{1}}(z)\left|f_{21}(z), \ldots, f_{2 m_{2}}(z)\right| \cdots \mid f_{d 1}(z), \ldots, f_{d m_{d}}(z)\right) .
$$

Also, for a holomorphic function $\varphi$ in $\mathbb{D}$, define an $m$-dimensional column vector $\boldsymbol{v}[\varphi]$ by

$$
\boldsymbol{v}[\varphi]={ }^{T}\left([\varphi]_{10}, \ldots,[\varphi]_{1, m_{1}-1}\left|[\varphi]_{20}, \ldots,[\varphi]_{2, m_{2}-1}\right| \cdots \mid[\varphi]_{d 0}, \ldots,[\varphi]_{d, m_{d}-1}\right),
$$

where $[\varphi]_{i j}$ is the $j$-th coefficient in the Taylor series expansion at $z=p_{i}$, i.e.,

$$
[\varphi]_{i j}=\left.\frac{1}{j !} \frac{d^{j} \varphi(z)}{d z^{j}}\right|_{z=p_{i}} .
$$

In addition, let 1 denote a constant vector $\boldsymbol{f}(0)=\boldsymbol{v}[1]={ }^{T}(1,0, \ldots, 0|1,0, \ldots, 0| \cdots \mid 1,0, \ldots, 0)$.

To obtain the main results of this note, let us prepare three lemmas.

Lemma 1. Let $n \geq n_{0}$. If $\varphi$ is a holomorphic function in $\mathbb{D} \cup \mathbb{T}$, then $\left(H_{n} \varphi\right)(z)$ is a linear combination of $f_{i j}^{\sharp}(z)$ expressed in the form

$$
\left(H_{n} \varphi\right)(z)={ }^{T} \boldsymbol{f}^{\sharp}(z) A B_{n} \boldsymbol{v}[\varphi],
$$

where $A$ and $B_{n}$ respectively are $d \times d$ block diagonal matrices with $m_{i} \times m_{i}$ submatrices

$$
A_{i}=\left(a_{i, j+k-1}\right) \quad \text { (upper anti-triangular Hankel matrices) }
$$

and

$$
\left.B_{n, i}=\left(\begin{array}{c}
n \\
j-k
\end{array}\right) p_{i}^{n-(j-k)}\right) \quad \text { (lower triangular Toeplitz matrices), }
$$

the displayed terms being the $(j, k)$-entries.

Proof. The value of the function $H_{n} \varphi$ can be evaluated by the formula

$$
\left(H_{n} \varphi\right)(z)=\frac{1}{2 \pi i} \int_{\mathbb{T}} \frac{\zeta^{n} h(\zeta) \varphi(\zeta)}{h^{\dagger}(\zeta)(z-\zeta)} d \zeta \quad(z \in \mathbb{C} \backslash(\mathbb{D} \cup \mathbb{T})) .
$$

In view of the partial fraction expansion (5), it is seen that the integrand is holomorphic in $\mathbb{D} \cup \mathbb{T}$ except for poles at $z=p_{1}, p_{2}, \ldots, p_{d}$ since the singularity at $z=0$ is removable for $n \geq n_{0}$. By Leibniz's rule,

$$
\begin{aligned}
\frac{1}{(r-1) !} \frac{d^{r-1}}{d \zeta^{r-1}} \frac{\zeta^{n} \varphi(\zeta)}{z-\zeta} & =\sum_{j=1}^{r} \frac{1}{(z-\zeta)^{j}} \cdot \frac{1}{(r-j) !} \frac{d^{r-j}}{d \zeta^{r-j}}\left\{\zeta^{n} \varphi(\zeta)\right\} \\
& =\sum_{j=1}^{r} \frac{1}{(z-\zeta)^{j}} \sum_{k=1}^{r-j+1}\left(\begin{array}{c}
n \\
r-j+1-k
\end{array}\right) \zeta^{n-(r-j+1-k)} \frac{1}{(k-1) !} \frac{d^{k-1}}{d \zeta^{k-1}} \varphi(\zeta) .
\end{aligned}
$$


Thus, by the formula (5) and the residue theorem,

$$
\begin{aligned}
\left(H_{n} \varphi\right)(z) & =\sum_{i=1}^{d} \sum_{r=1}^{m_{i}} a_{i r} \cdot \frac{1}{2 \pi i} \int_{\mathbb{T}} \frac{\zeta^{n} \varphi(\zeta)}{\left(\zeta-p_{i}\right)^{r}(z-\zeta)} d \zeta \\
& =\sum_{i=1}^{d} \sum_{r=1}^{m_{i}} a_{i r} \sum_{j=1}^{r} \frac{1}{\left(z-p_{i}\right)^{j}} \sum_{k=1}^{r-j+1}\left(\begin{array}{c}
n \\
r-j+1-k
\end{array}\right) p_{i}^{n-(r-j+1-k)}[\varphi]_{i, k-1} \\
& =\sum_{i=1}^{d} \sum_{j=1}^{m_{i}} \frac{1}{\left(z-p_{i}\right)^{j}} \sum_{k=1}^{m_{i}-j+1}\left\{\sum_{l=k}^{m_{i}-j+1} a_{i, j+l-1}\left(\begin{array}{c}
n \\
l-k
\end{array}\right) p_{i}^{n-(l-k)}\right\}[\varphi]_{i, k-1},
\end{aligned}
$$

in which $l=r-j+1$, and this may be rewritten into the matrix form of the assertion.

Lemma 2. Let $\boldsymbol{u}$ be an m-dimensional column vector. If $\varphi(z)={ }^{T} \boldsymbol{f}(z) \boldsymbol{u}$, then

$$
\boldsymbol{v}[\varphi]=C \boldsymbol{u},
$$

where $C$ is a $d \times d$ block matrix with $m_{i} \times m_{j}$ submatrices

$$
C_{i j}=\left(\left[f_{j l}\right]_{i, k-1}\right),
$$

the displayed term being the $(k, l)$-entry. The value of $\left[f_{j l}\right]_{i k}$ can be evaluated as

$$
\left[f_{j l}\right]_{i k}=\sum_{r=0}^{k}\left(\begin{array}{c}
k \\
r
\end{array}\right)\left(\begin{array}{c}
l+k-r-1 \\
k
\end{array}\right) \frac{p_{i}^{l-1-r} \bar{p}_{j}^{k-r}}{\left(1-p_{i} \bar{p}_{j}\right)^{l+k-r}} .
$$

Proof. This assertion is merely a matrix representation of the linear equations

$$
[\varphi]_{i k}=\sum_{j=1}^{d} \sum_{l=1}^{m_{j}}\left[f_{j l}\right]_{i k} u_{j l},
$$

subject to $\boldsymbol{u}={ }^{T}\left(u_{11}, \ldots, u_{1 m_{1}}\left|u_{21}, \ldots, u_{2 m_{2}}\right| \cdots \mid u_{d 1}, \ldots, u_{d m_{d}}\right)$.

Lemma 3. If $n \geq n_{0}$, then

$$
\left\{\left(I-H_{n}^{*} H_{n}\right)^{-1} 1\right\}(z)=1+{ }^{T} \boldsymbol{f}(z) \overline{A B}_{n} G_{n}\left(I-\bar{G}_{n} G_{n}\right)^{-1} \mathbf{1},
$$

where

$$
G_{n}=\bar{C} A B_{n}
$$

In particular, this gives

$$
\boldsymbol{v}\left[\left(I-H_{n}^{*} H_{n}\right)^{-1} 1\right]=\left(I-\bar{G}_{n} G_{n}\right)^{-1} \mathbf{1} .
$$

Proof. Assume $n \geq n_{0}$. Let $\boldsymbol{u}$ be an $m$-dimensional column vector. It follows from Lemmas 1 and 2 that, for $\varphi(z)={ }^{T} \boldsymbol{f}(z) \boldsymbol{u}$,

$$
\left(H_{n} \varphi\right)(z)={ }^{T} \boldsymbol{f}^{\sharp}(z) A B_{n} C \boldsymbol{u} .
$$

Since $\left(H_{n}^{*} \psi\right)(z)=\left(H_{n} \psi^{\sharp}\right)^{\sharp}(z)$, this implies that, for $\psi(z)={ }^{T} \boldsymbol{f}^{\sharp}(z) \boldsymbol{u}$,

$$
\left(H_{n}^{*} \psi\right)(z)={ }^{T} \boldsymbol{f}(z) \overline{A B_{n} C} \boldsymbol{u} .
$$

Clearly, $I-\bar{G}_{n} G_{n}$ is invertible if and only if $I-G_{n} \bar{G}_{n}$ is invertible. To check the latter, let $\left(I-G_{n} \bar{G}_{n}\right) \boldsymbol{u}=\mathbf{0}$. By applying the above results to $\chi(z)={ }^{T} \boldsymbol{f}(z) \overline{A B}_{n} \boldsymbol{u}$,

$$
\left\{\left(I-H_{n}^{*} H_{n}\right) \chi\right\}(z)={ }^{T} \boldsymbol{f}(z) \overline{A B}_{n}\left(I-G_{n} \bar{G}_{n}\right) \boldsymbol{u}=0 .
$$


Since $I-H_{n}^{*} H_{n}$ is invertible, one has $\chi(z)=0$, so that $\overline{A B}_{n} \boldsymbol{u}=\mathbf{0}$. Also, $\overline{A B}_{n}$ is invertible, as is seen from

$$
\operatorname{det}\left(A_{i}\right)=\operatorname{sgn}(\sigma)\left(a_{i, m_{i}}\right)^{m_{i}} \neq 0, \quad \operatorname{det}\left(B_{i, n}\right)=\left(p_{i}^{n}\right)^{m_{i}} \neq 0,
$$

where $\sigma$ is a permutation defined by $\sigma(k)=m_{i}-k+1\left(k=1,2, \ldots, m_{i}\right)$. Hence, $\boldsymbol{u}=\mathbf{0}$, and $I-G_{n} \bar{G}_{n}$ is invertible. Now, by using Lemma 1 and the above results,

$$
\begin{aligned}
\left\{H_{n}^{*} H_{n}\left(1+{ }^{T} \boldsymbol{f} \overline{A B}_{n} G_{n}\left(I-\bar{G}_{n} G_{n}\right)^{-1} \mathbf{1}\right)\right\}(z) & =\left\{{ }^{T} \boldsymbol{f} \overline{A B}_{n} G_{n}\left(I+\bar{G}_{n} G_{n}\left(I-\bar{G}_{n} G_{n}\right)^{-1}\right) \mathbf{1}\right\}(z) \\
& =\left\{{ }^{T} \boldsymbol{f} \overline{A B}_{n} G_{n}\left(I-\bar{G}_{n} G_{n}\right)^{-1} \mathbf{1}\right\}(z),
\end{aligned}
$$

which gives

$$
\left\{\left(I-H_{n}^{*} H_{n}\right)\left(1+{ }^{T} \boldsymbol{f} \overline{A B}_{n} G_{n}\left(I-\bar{G}_{n} G_{n}\right)^{-1} \mathbf{1}\right)\right\}(z)=1 .
$$

Again, since $I-H_{n}^{*} H_{n}$ is invertible, the first half of the assertion holds. The other half can be shown by applying Lemma 2 to $\left\{\left(I-H_{n}^{*} H_{n}\right)^{-1} 1\right\}(z)=1+{ }^{T} \boldsymbol{f}(z) \boldsymbol{u}$ with $\boldsymbol{u}=\overline{A B}_{n} G_{n}\left(I-\bar{G}_{n} G_{n}\right)^{-1} \mathbf{1}$, and then doing a calculation similar to the above one.

The following result presents an explicit matrix representation of a discrete analog of Seghier's prediction formula in [10, Corollary 2]. (The original result implicitly gives the "form" of the formula under some general assumption.)

Theorem 4. For $n \geq n_{0}$, the monic orthogonal polynomials are expressed as

$$
\Phi_{n}(z)=z^{n} \frac{h(0)}{h^{\dagger}(z)}\left(1+{ }^{T} \boldsymbol{f}(z) \overline{A B}_{n} G_{n}\left(I-\bar{G}_{n} G_{n}\right)^{-1} \mathbf{1}\right)-\frac{h(0)}{h(z)}{ }^{T} \boldsymbol{f}^{\sharp}(z) A B_{n}\left(I-\bar{G}_{n} G_{n}\right)^{-1} \mathbf{1},
$$

and their squared norms admit the representation

$$
\left\|\Phi_{n}\right\|_{\mu}^{2}=h(0)^{2}\left(1+{ }^{T} \boldsymbol{g}_{n}\left(I-\bar{G}_{n} G_{n}\right)^{-1} \bar{G}_{n} \mathbf{1}\right),
$$

where

$$
\boldsymbol{g}_{n}=A B_{n} \mathbf{1}
$$

Proof. The first formula is readily obtained by applying Lemmas 1 and 3 to Seghier's formula (4). To prove the other one, note that $\left\|\Phi_{n}\right\|_{\mu}^{2}=\left(\Phi_{n}, z^{n}\right)_{\mu}$, for which Seghier's formula (4) gives

$$
\frac{\Phi_{n}(z) w(z)}{z^{n}}=h(0) h(z)\left\{\left(I-H_{n}^{*} H_{n}\right)^{-1} 1\right\}(z)-\frac{h(0) h^{\dagger}(z)}{z^{n}}\left\{H_{n}\left(I-H_{n}^{*} H_{n}\right)^{-1} 1\right\}(z) .
$$

Since the second term of the RHS is holomorphic in $\mathbb{T} \cup \mathbb{E}$ and vanishes at $z=\infty$,

$$
\begin{aligned}
\left\|\Phi_{n}\right\|_{\mu}^{2} & =\frac{1}{2 \pi i} \int_{\mathbb{T}} \frac{\Phi_{n}(\zeta) w(\zeta)}{\zeta^{n}} \frac{d \zeta}{\zeta}=h(0) \cdot \frac{1}{2 \pi i} \int_{\mathbb{T}} \frac{h(\zeta)\left\{\left(I-H_{n}^{*} H_{n}\right)^{-1} 1\right\}(\zeta)}{\zeta} d \zeta \\
& =h(0)^{2}\left\{\left(I-H_{n}^{*} H_{n}\right)^{-1} 1\right\}(0) .
\end{aligned}
$$

Observed that ${ }^{T}\left(A B_{n}\right)=A B_{n}$. By Lemma 3 and $\bar{G}_{n}\left(I-G_{n} \bar{G}_{n}\right)^{-1}=\left(I-\bar{G}_{n} G_{n}\right)^{-1} \bar{G}_{n}$,

$$
\begin{aligned}
\left\{\left(I-H_{n}^{*} H_{n}\right)^{-1} 1\right\}(0) & =\overline{\left\{\left(I-H_{n}^{*} H_{n}\right)^{-1} 1\right\}(0)}=1+{ }^{T} \mathbf{1} A B_{n} \bar{G}_{n}\left(I-G_{n} \bar{G}_{n}\right)^{-1} \mathbf{1} \\
& =1+{ }^{T} \boldsymbol{g}_{n}\left(I-\bar{G}_{n} G_{n}\right)^{-1} \bar{G}_{n} \mathbf{1},
\end{aligned}
$$

which completes the proof.

The following matrix representation is particularly simple.

Theorem 5. For $n>n_{0}$, the Verblunsky coefficients admit the representation

$$
\alpha_{n}={ }^{T} \boldsymbol{g}_{n-1}\left(I-\bar{G}_{n} G_{n}\right)^{-1} \mathbf{1} .
$$


Proof. Seghier's formula (4) shows that

$$
\frac{h(z) \Phi_{n}(z)}{h(0)}=\frac{z^{n} h(z)}{h^{\dagger}(z)}\left\{\left(I-H_{n}^{*} H_{n}\right)^{-1} 1\right\}(z)-\left\{H_{n}\left(I-H_{n}^{*} H_{n}\right)^{-1} 1\right\}(z),
$$

in which the last term, i.e., $\left\{H_{n}\left(I-H_{n}^{*} H_{n}\right)^{-1} 1\right\}(z)$, is holomorphic in $\mathbb{T} \cup \mathbb{E}$ and vanishes at $z=\infty$. Hence, by definition of the Verblunsky coefficients,

$$
\alpha_{n}=\Phi_{n}(0)=\frac{h(0) \Phi_{n}(0)}{h(0)}=\frac{1}{2 \pi i} \int_{\mathbb{T}} \frac{h(\zeta) \Phi_{n}(\zeta)}{h(0)} \frac{d \zeta}{\zeta}=\frac{1}{2 \pi i} \int_{\mathbb{T}} \frac{\zeta^{n-1} h(\zeta)}{h^{\dagger}(\zeta)}\left\{\left(I-H_{n}^{*} H_{n}\right)^{-1} 1\right\}(\zeta) d \zeta .
$$

Now, let $\varphi$ be a holomorphic function in $\mathbb{D} \cup \mathbb{T}$. By Leibniz's rule,

$$
\frac{1}{(r-1) !} \frac{d^{r-1}}{d \zeta^{r-1}}\left\{\zeta^{n-1} \varphi(\zeta)\right\}=\sum_{j=1}^{r}\left(\begin{array}{l}
n-1 \\
r-j
\end{array}\right) \zeta^{(n-1)-(r-j)} \cdot \frac{1}{(j-1) !} \frac{d^{j-1}}{d \zeta^{j-1}} \varphi(\zeta) .
$$

So, as in the proof of Lemma 1, the formula (5) and the residue theorem imply that, for $n>n_{0}$,

$$
\begin{aligned}
\frac{1}{2 \pi i} \int_{\mathbb{T}} \frac{\zeta^{n-1} h(\zeta) \varphi(\zeta)}{h^{\dagger}(\zeta)} d \zeta & =\sum_{i=1}^{d} \sum_{r=1}^{m_{i}} a_{i r} \cdot \frac{1}{2 \pi i} \int_{\mathbb{T}} \frac{\zeta^{n-1} \varphi(\zeta)}{\left(\zeta-p_{i}\right)^{r}} d \zeta \\
& =\sum_{i=1}^{d} \sum_{r=1}^{m_{i}} a_{i r} \sum_{j=1}^{r}\left(\begin{array}{c}
n-1 \\
r-j
\end{array}\right) p_{i}^{(n-1)-(r-j)}[\varphi]_{i, j-1} \\
& =\sum_{i=1}^{d} \sum_{j=1}^{m_{i}}\left\{\sum_{k=1}^{m_{i}-j+1} a_{i, j+k-1}\left(\begin{array}{l}
n-1 \\
k-1
\end{array}\right) p_{i}^{(n-1)-(k-1)}\right\}[\varphi]_{i, j-1},
\end{aligned}
$$

in which $k=r-j+1$, and this may be expressed as ${ }^{T} \boldsymbol{g}_{n-1} \boldsymbol{v}[\varphi]$. So, Lemma 3 gives the representation.

Remark 6. If $m=d$, i.e., if $P$ has only simple zeros, the above results take simple forms without block structure. Indeed, the basic three matrices are written as

$$
A=\operatorname{diag}\left(a_{1}, \ldots, a_{d}\right), \quad B_{n}=\operatorname{diag}\left(p_{1}^{n}, \ldots, p_{d}^{n}\right), \quad C=\left(\begin{array}{ccc}
\frac{1}{1-p_{1} \bar{p}_{1}} & \cdots & \frac{1}{1-p_{1} \bar{p}_{d}} \\
\vdots & & \vdots \\
\frac{1}{1-p_{d} \bar{p}_{1}} & \cdots & \frac{1}{1-p_{d} \bar{p}_{d}}
\end{array}\right)
$$

where $a_{i}=a_{i 1}$, the residue of $h / h^{\dagger}$ at its pole $z=p_{i}$.

\section{Acknowledgement}

The authors would like to thank the referee for helpful comments on the presentation of this note.

\section{References}

[1] N. H. Bingham, A. Inoue and Y. Kasahara, An explicit representation of Verblunsky coefficients, Statist. Probab. Lett. 82 (2012) 403-410.

[2] P. J. Brockwell and R. A. Davis, Time series: theory and methods, Second edition, Springer-Verlag, New York, 1991.

[3] A. Inoue, Asymptotics for the partial autocorrelation function of a stationary process, J. Anal. Math. 81 (2000) 65-109.

[4] A. Inoue, Asymptotic behavior for partial autocorrelation functions of fractional ARIMA processes, Ann. Appl. Probab. 12 (2002) 1471-1491.

[5] A. Inoue, AR and MA representation of partial autocorrelation functions, with applications, Probab. Theory Related Fields 140 (2008) 523-551.

[6] A. Inoue and Y. Kasahara, Partial autocorrelation functions of the fractional ARIMA processes with negative degree of differencing, J. Multivariate Anal. 89 (2004) 135-147. 
[7] A. Inoue and Y. Kasahara, Explicit representation of finite predictor coefficients and its applications, Ann. Statist. 34 (2006) 973-993.

[8] Y. Kasahara and N.H. Bingham, Verblunsky coefficients and Nehari sequences, Trans. Amer. Math. Soc. 366 (2014) $1363-1378$.

[9] V. V. Peller, Hankel operators and their applications, Springer-Verlag, New York, 2003.

[10] A. Seghier, Prédiction d'un processus stationnaire du second ordre de covariance connue sur un intervalle fini, Illinois J. Math. 22 (1978) 389-401.

[11] B. Simon, Orthogonal polynomials on the unit circle, Part 1. Classical theory, American Mathematical Society, Providence, RI, 2005.

[12] K. Yabuta, Some uniqueness theorems for $H^{p}\left(U^{n}\right)$ functions, Tôhoku Math. J. 24 (1972), 353-357. 